\title{
A Commentary on Attitudes Towards Deep Brain Stimulation for Addiction
}

\author{
Karen E. Lee, Mahendra T. Bhati, Casey H. Halpern \\ Department of Neurosurgery, Stanford University 300 Pasteur Dr., Edwards Bldg. Stanford, CA 94305, USA
}

\section{Article Info}

\section{Article Notes}

Received: October 20, 2016

Accepted: November 15, 2016

*Correspondence:

Dr. Casey H. Halpern, Department of Neurosurgery, Stanford University 300 Pasteur Dr., Edwards Bldg. Stanford, CA 94305 , USA, Email: chalpern@stanford.edu

(C) 2016 Halpern $\mathrm{CH}$. This article is distributed under the terms of the Creative Commons Attribution 4.0 International License

\section{Keywords:}

DBS

addiction

ethics

patient autonomy

informed consent

clinical trials

\section{ABSTRACT}

Deep brain stimulation (DBS) has proven to be an effective treatment for neurologic disorders such as Parkinson's disease, and is currently being investigated as a therapy for psychiatric diseases such as addiction, major depressive disorder, and obsessive compulsive disorder. In this commentary, we review and discuss the findings presented in the Letter to the Editor entitled "Attitudes towards treating addiction with deep brain stimulation," written by Ali et $\mathrm{al}^{1}$. The survey presented in this Letter reported general approval for examining the effects of DBS on addictive disorders in a clinical trial, but highlighted critical areas of concern including informed consent, patient autonomy, appropriate medical practice, passing of clinical trial milestones, and implications on law enforcement.

\section{Introduction}

Since the inception of its explorative use in the 1960s and subsequent approval by the U.S. Food and Drug Administration for tremor disorders in 2002, deep brain stimulation (DBS) has demonstrated tremendous therapeutic potential in a number of neurologic and psychiatric disorders ${ }^{2,3}$. DBS has expanded to a number of other conditions such as obsessive compulsive disorder, and is currently being studied in clinical trials for other neurologic and psychiatric disorders ${ }^{4}$. Most recently, DBS has emerged as a promising treatment option for addictive disorders ${ }^{5}$, which continues to be a major public health concern with loss of control over various substances and high relapse rates compounded by looming medical and associated socioeconomic costs 6 . Current treatments are few and suboptimal, thus necessitating a therapy that can directly intervene on neural circuits underlying addiction.

As an implantable brain interfacing device, DBS as a treatment for addictive disorders presents an alternative from available pharmacotherapy. A neurosurgical procedure is required to implant the DBS system to deliver electrical stimulation to an identified critical structure in the brain. The nucleus accumbens, a brain region reported to modulate reward-based decision making, is a potential target for treating addiction and one that has been repeatedly supported in preclinical studies for DBS ${ }^{4}$. However, the development and implementation of this intervention has compelling ethical challenges ${ }^{4,8}$. Here, we review a Letter to the Editor entitled "Attitudes Towards Treating Addiction with Deep Brain Stimulation," written by Ali et al. This study demonstrated general support for 
initiating a clinical trial to examine DBS as a potential treatment of addiction. However, there were some critical areas of concern including clinical trial design, managing side effects, patient postoperative follow-ups. Through this commentary, we discuss how DBS may influence the future treatment of addiction and the important ramifications of this novel therapeutic development.

\section{Patient autonomy}

A major theme presented in this Letter was the notion of patient autonomy. For a surgical procedure that modifies functions and traits relating to a person's identity, this is an important ethical cornerstone to consider. Within the last three decades, respect for patient autonomy and self-determination have taken center stage in health policy and bioethics ${ }^{9}$. With the goals of supporting individual values and rights, autonomy encompasses principles such as informing patients of various treatment options and disclosing medical information for sound decision-making ${ }^{10}$. Furthermore, the shift from physician paternalism to patient self-determination was a major victory in the world of American bioethics. In the context of targeting critical regions, the way in which an addiction compromises cognitive capacities and generates patient vulnerabilities creates difficulties in respecting autonomy. This, compounded by current trend in bioethics, are the two major obstacles in the battlefield of DBS for addicted patients. Nevertheless, the cognitive and psychiatric comorbidities of addiction prevent subjects from acting as autonomous agents because the disease coerces their behavior ${ }^{11}$. However, DBS for addiction can be justified if the treatment can in fact restore autonomy by restoring cognitive competency to make choices free from compulsions. Future studies on how DBS interacts with a patient's decision-making ability, particularly in the context of their own health, will be important to pursue.

\section{Financial incentives}

The survey results revealed a critical area of concern, namely the allure of financial incentives as they can breach the integrity of informed consent. The heightened vulnerability of addiction patients could make them more willing to consent due to desperate hope for recovery. Nonetheless, the argument for temporarily infringing on patient autonomy to create patient autonomy still holds since removing the medical and social costs of addiction is a superior aim than maintaining them. Two solutions to managing the issue of financial incentives and therapeutic misconceptions exist. First, physicians should provide additional support to enable patients to realize the full spectrum of risks and benefits so that unrealistic expectations do not compromise patient autonomy. A protocol should exist that evaluates the patient's expectations from a multidisciplinary perspective in selecting candidates for DBS. Second, the financial incentive should be kept at a sufficiently low value to optimize informed consent and prevent financial gain.

DBS and the relationship between brain and moral agency in the context of crime

Responsibility under the law after DBS was discussed in this Letter, thus illuminating the need for an evolved view of legal practice in the context of this surgical procedure. When considering the application of autonomy to DBS, two subtly distinct definitions should be noted. First, autonomy is the willingness to consent to medical procedures, as discussed previously. Second, however, is the ability to lead an autonomous life without a sense of alienation of self ${ }^{12}$. Indeed, DBS may have a profound influence on behavior and thus self, with reports of changes in mood and personality so drastic that roles between the patient and those they have close relationship change ${ }^{13}$. These changes could theoretically make patients not possess the relevant capacities of judgment and self-control. However, recent data from a study on DBS of the accumbens suggested that there were no associations between this intervention and cognitive changes ${ }^{14}$.

\section{Implications on clinical practice}

The traditional goal of medicine is to restore normal functioning through relief of suffering. This remains to be tested in clinical trials but is the goal of developing this novel therapy. If DBS relieves the symptoms of addiction while also enhancing memory or mood, should treatment continue? Currently, clinical trials for DBS are being pursued, and thus passing milestones at each progressive stage would provide opportunity for continual evaluation and safe usage. These milestones would include DBS device testing and stimulation parameter optimization in preclinical models, investigational device exemption application to the US FDA, submission of clinical study design to the IRB, multidisciplinary patient screening, milestone driven studies with strict go-no go criteria, followed by final data analysis by an objective expert in addition to the research team.

\section{Conclusions}

Despite the expanding use of DBS for addiction, there is a lack of clarity concerning ethical questions of legal responsibility and the role of the physician-patient relationship in dictating medical treatment. Moreover, changes in personal identity can lead to undesirable behavior and in doing so, infringe upon patient-autonomy, and complicate delivery of treatment. The fact that the mechanism by which DBS works remains unknown means there should be general caution towards whether the procedure is in fact reversible, as this could have profound and lasting effects on the decision-making 
capacity of patients ${ }^{15}$. A multidisciplinary team to have proper oversight mechanisms in place to handle changes in mental competence and informed consent is necessary. In addition, safeguards should exist to handle the unique problem of vulnerability in this addicted population of patients towards financial incentives. Given that DBS will not be limited to patients with addictive disorders, these considerations will be important for framing future research and design of clinical trials.

\section{Acknowledgments}

This effort was supported in part by funding from the National Institute of Health (K12NS080223).

\section{References}

1. Ali R, DiFrancesco MF, Ho AL, Kampman KM, Caplan AL, Halpern CH. Attitudes towards treating addiction with deep brain stimulation. Brain Stimulation. 2016 May-Jun;9(3):466-8. doi: 10.1016/j. brs.2016.03.009. Epub 2016 Mar 15.

2. Rossi PJ, Gunduz A, Judy J, et al. Proceedings of the Third Annual Deep Brain Stimulation Think Tank: A Review of Emerging Issues and Technologies. Frontiers in Neuroscience. 2016;10:119. doi:10.3389/ fnins.2016.00119.

3. Okun MS, Foote KD. Parkinson's disease DBS: what, when, who and why? The time has come to tailor DBS targets. Expert review of neurotherapeutics. 2010;10(12):1847-1857.

4. Halpern $\mathrm{CH}$, Tekriwal A, Santollo J, et al. Amelioration of binge eating by nucleus accumbens shell deep brain stimulation in mice involves D2 receptor modulation. The Journal of neuroscience: the official journal of the Society for Neuroscience. 2013;33(17):7122-7129. doi:10.1523/JNEUROSCI.3237-12.2013.
5. Halpern C.H., Torres N., Hurtig H.I., Wolf J.A., Stephen J., Oh M.Y., Williams N.N., Dichter M.A., Jaggi J.L., Caplan A.L., et al. Expanding applications of deep brain stimulation: A potential therapeutic role in obesity and addiction management. Acta Neurochir. 2011;153:22932306. doi: $10.1007 /$ s00701-011-1166-3.

6. Stephen $\mathrm{JH}$, Halpern $\mathrm{CH}$, Barrios $\mathrm{CJ}$, Balmuri U, Pisapia JM, Wolf JA et al. Deep brain stimulation compared with methadone maintenance for the treatment of heroin dependence: a threshold and costeffectiveness analysis. Addiction. 2011;107:624-634.

7. Muller UJ, et al. Deep brain stimulation of the nucleus accumbens for the treatment of addiction.Ann N Y Acad Sci. 2013;1282:119-128.

8. Beauchamp T.L., Childress J.F. (2001), Principles of Biomedical Ethics (5th ed). Oxford University Press, New York

9. Caplan, A. (2006). Ethical issues surrounding forced, mandated, or coerced treatment. Journal of Sbustance Abuse Treatment, 31, 117120.

10. Caplan, A. (2008). Denying autonomy in order to create it: the paradox of forcing treatment upon addicts. Addiction, 103, 1919-1921.

11. Kraemer, F. 2013. Me, myself and my brain implant: Deep brain stimulation raises questions of personal authenticity and alienation. Neuroethics 6: 483-97.

12. Witt K, Kuhn J, Timmermann L, Zurowski M, Woopen C. Deep Brain Stimulation and the Search for Identity. Neuroethics. 2013;6(3):499511. doi:10.1007/s12152-011-9100-1.

13. Kubu CS, et al. Cognitive outcome after ventral capsule/ventral striatum stimulation for treatment-resistant major depression. J Neurol Neurosurg Psychiatry. doi:10.1136/jnnp-2016-313803

14. McIntyre CC, Anderson RW. Deep brain stimulation: the control of network activity via neurochemistry modulation . J Neurochem. 2016 Oct;139 Suppl 1:338-345. doi: 10.1111/jnc.13649. Epub 2016 Jun 8. 